\title{
Active Targeted Macrophage-mediated Delivery of Catalase to Affected Brain Regions in Models of Parkinson's Disease
}

Yuling Zhao ${ }^{1,2}$, Matthew J. Haney ${ }^{1,2}$, Vivek Mahajan ${ }^{1,3}$, Benjamin C. Reiner ${ }^{4}$ Anna Dunaevsky ${ }^{4}$, R. Lee Mosley ${ }^{1,5,6}$, Alexander V. Kabanov ${ }^{1,2}$, Howard E. Gendelman ${ }^{1,2,5,6}$ and Elena V. Batrakova ${ }^{1,2 *}$

${ }^{1}$ Center for Drug Delivery and Nanomedicine, University of Nebraska Medical Center, Omaha, Nebraska, USA

${ }^{2}$ Department of Pharmaceutical Sciences, University of Nebraska Medical Center, Omaha, Nebraska, USA

${ }^{3}$ Department of pathology and microbiology, University of Nebraska Medical Center, Omaha, Nebraska, USA

${ }^{4}$ Developmental Neuroscience, Munroe-Meyer Institute, University of Nebraska Medical Center, Omaha, Nebraska, USA

${ }^{5}$ Center for Neurovirology and Neurodegenerative Disorders, University of Nebraska Medical Center, Omaha, Nebraska, USA

${ }^{6}$ Department of Pharmacology and Experimental Neuroscience, University of Nebraska Medical Center, Omaha, Nebraska, USA

\begin{abstract}
We previously demonstrated that monocyte-macrophage based drug delivery can be applied to a spectrum of infectious, neoplastic, and degenerative disorders. In particular, bone marrow-derived macrophages (BMM) loaded with nano formulated catalase, "nanozyme", were shown to attenuate neuro inflammation and nigrostriatal degeneration in rodent models of Parkinson's disease (PD). Nonetheless, the pharmacokinetics and biodistribution of BMMincorporated nanozyme has not been explored. To this end, we now demonstrate that BMM, serving as a "depot" for nanozyme, increased area under the curve(AUC), half-life, and mean residence time in blood circulation of the protein when compared to the nanozyme administered alone. Accordingly, bioavailability of the nanozyme for the brain, spleen, kidney, and liver was substantially increased. Importantly, nanozyme-loaded BMM targeted diseased sites and improved transport across the blood brain barrier. This was seen specifically in affected brain subregions in models of PD. Engaging natural immune cells such as monocyte-macrophages as drug carriers provides a new perspective for therapeutic delivery for PD and also likely a range of other inflammatory and degenerative diseases.
\end{abstract}

Keywords: Drug biodistribution; Cell-mediated drug delivery, Nanozyme; Parkinson's disease; Pharmacokinetics; Targeted delivery

Abbreviations: AUC: Area under the curve; BBB: Blood brain barrier; BMM: Bone marrow-derived macrophages; CL: Clearance; $t_{1 / 2}$ : Effective elimination half-life; i.t.v.: Intratail vein; i.j.v.: Intrajugular vein; intraperitoneal injections, i.p; 6-OHDA: 6-hydroxidophamine; LPS: lipopolysaccharides; MPTP: 1-methyl-4-phenyl-1,2,3,6tetrahydro-pyridine; MRT: Mean residence time; PD: Parkinson's disease; PBS: Phosphate saline buffer; PEI-PEG: Poly(ethyleneimine)poly(ethylene glycol); SNpc: Substantianigra pars compacta,.

\section{Introduction}

The development of targeted drug delivery will improve therapeutic efficacy through reductions in drug dosing intervals, and diminished toxicities. Previously, were ported that substantive decreases in neuro inflammation and nigrostrital degeneration were achieved in mouse models of Parkinson's disease (PD) when bone marrow-derived macrophages (BMM)were used as carriers for the antioxidant enzyme, catalase to the brain $[1,2]$. BMM can carry significant amounts of the enzyme then slowly release it in active form [1]. Importantly, the packaging of catalase into a block ionomer complex with a cationic block copolymer, poly (ethyleneimine)-poly (ethylene glycol), and "nanozyme" precludes macrophage-mediated enzyme degradation enabling drug-carried enzyme stability. The functional activities of the released from BMM catalase were demonstrated in its ability to decompose microglial hydrogen peroxide. This was produced in pathophysiologic conditions reflective of disease as follows nitrated alpha-synuclein or tumor necrosis factor alpha (TNF-a)-induced microglial activation [1]. Parallel investigations demonstrated that catalase nanozyme composition and its physicochemical characteristics (morphology, size, and $\xi$-potential) were predictors of macrophage loading, release, and enzymatic activities [3].

For the mechanisms of improved therapeutic endpoints by macrophage carried nanozyme, three possible pathways are suggested: 1) that nanozyme-loaded BMM can cross the blood brain barrier (BBB) and release catalase at sites of inflammation and disease, the substantianigra pars compacta, $S N p c ; 2)$ an enzyme "depot" is established such that nanozyme is released slowly from BMM to the blood stream and then crosses the BBB; and 3) catalase nanozyme released from BMM in the liver and spleen suppresses peripheral leukocyte activation that results in significant secondary nigrostriatal protection [2].

To determine the mechanism by which the nanozyme could exert its therapeutic outcome, we evaluated how loading the drug to cellcarriers affects its biodistribution and transport across the BBB in different models of PD. The data demonstrate that BMM provided targeted nanozyme delivery to sites of inflammation by increasing drug transport across the BBB and brain availability for catalase.

\section{Materials and Methods}

\section{Reagents}

Catalase from bovine liver erythrocytes was provided by Calbiochem (San Diego, CA, USA).1-methyl-4-phenyl-1,2,3,6-

*Corresponding author: Elena V. Batrakova, Center for Drug Delivery and Nanomedicine, 985830 Nebraska Medical Center, Omaha, NE 68198-5830; Tel: (402) 559-9364 ; Fax (402) 559-9365; Email: ebatrako@unmc.edu

Received July 30, 2011; Accepted September 07, 2011; Published September 10, 2011

Citation: Zhao Y, Haney MJ, Mahajan V, Reiner BC, Dunaevsky A, et al. (2011) Active Targeted Macrophage-mediated Delivery of Catalase to Affected Brain Regions in Models of Parkinson's Disease. J Nanomedic Nanotechnol S4:003. doi:10.4172/2157-7439.S4-003

Copyright: (c) 2011 Zhao Y, et al. This is an open-access article distributed under the terms of the Creative Commons Attribution License, which permits unrestricted use, distribution, and reproduction in any medium, provided the original author and source are credited. 
Citation: Zhao Y, Haney MJ, Mahajan V, Reiner BC, Dunaevsky A, et al. (2011) Active Targeted Macrophage-mediated Delivery of Catalase to Affected Brain Regions in Models of Parkinson's Disease. J Nanomedic Nanotechnol S4:003. doi:10.4172/2157-7439.S4-003

Page 2 of 8

tetrahydro-pyridine(MPTP), 6-hydroxidophamine (6-OHDA), lipopolysaccharides (LPS), Triton X-100, and Sephadex G-25 were purchased from Sigma-Aldrich (St. Louis, MO, USA). Methoxypolyethylene glycol epoxy (Me-PEG-epoxy) was obtained from Shearwater Polymer Inc. (Huntsville, AL, USA). Poly (ethyleneimine)poly (ethylene glycol) (PEI (2K)-PEG (10K)) was synthesized as described previously [1] by conjugation of PEI and Me-PEG-epoxy. ${ }^{3} \mathrm{H}$-labeled catalase was custom-synthesized by PerkinElmer Life (Boston, MA, USA). Alexa Fluor 680 Protein Dye Kit was purchased from Molecular Probes, Eugene, OR.

\section{Animals}

Male C57BL/6 mice (Charles River Laboratories, USA) 6-8 weeks of age were used in pharmacokinetics, biodistribution and in vivo IVIS and epifluorescence microscopy imaging experiments. The animals were kept five per cage with an air filter cover under light- (12-hours light/dark cycle) and temperature-controlled $\left(22 \pm 1^{\circ} \mathrm{C}\right)$ environment. All manipulations with the animals were performed under a sterilized laminar hood. Food and water were given ad libitum. The animals were treated in accordance to the Principles of Animal Care outlined by National Institutes of Health and approved by the Institutional Animal Care and Use Committee of the University of Nebraska Medical Center.

\section{Cells}

Bone marrow-derived macrophages (BMM) were extracted from femurs of $\mathrm{C} 57 \mathrm{BL} / 6$ male mice according to previously published protocols [4] and cultured in a humidified incubator at $37^{\circ} \mathrm{C}$ and $5 \%$ $\mathrm{CO}_{2}$ for 10 days in DMEM medium (Invitrogen, Carlsbad, CA, USA) supplemented with $1,000 \mathrm{U} / \mathrm{mL}$ macrophage colony-stimulating factor (MCSF), a generous gift from Wyeth Pharmaceutical, Cambridge, MA, USA).

\section{Catalase nanozyme}

The polycomplex of catalase and PEI-PEG (nanozyme) was produced by mixing catalase and a block copolymer, PEI-PEG, which bind electrostatically to each other and form nanoparticles with an enzyme-polyion complex core and PEG corona [1,2]. Catalase and PEI-PEG were separately dissolved in phosphate-buffered saline (PBS) at room temperature. Pre-calculated volumes of the block copolymer solution were added drop-by-drop to the enzyme solution to achieve the desired charge ratio $(Z=1)$ that was calculated by dividing the amount of amino groups in the block copolymer protonated at $\mathrm{P}^{\mathrm{H}} 7.4$ [5] by the total amount of Gln and Asp in catalase. To obtain a narrow size distribution of the particles, the nanozymes were filtered through a $100 \mathrm{~nm}$ filter before use.

\section{Loading of BMM with nanozyme}

BMM grown on T75 flasks (1-2 x $10^{6}$ cells/flask) [6,7] were preincubated with assay buffer $(122 \mathrm{mM} \mathrm{NaCl}, 25 \mathrm{mM} \mathrm{NaHCO}, 10 \mathrm{mM}$ glucose, $3 \mathrm{mM} \mathrm{KCl}, 1.2 \mathrm{mM} \mathrm{MgSO}_{4}, 0.4 \mathrm{mM} \mathrm{K}_{2} \mathrm{HPO}_{4}, 1.4 \mathrm{mM} \mathrm{CaCl}_{2}$ and $10 \mathrm{mM}$ HEPES) for $20 \mathrm{~min}$. Following pre-incubation, the cells were supplemented with the nanozyme $(1 \mathrm{mg} / \mathrm{ml})$ for one hour at $37^{\circ} \mathrm{C}$. After the incubation, the cells were washed three times with ice-cold PBS and used in further experiments. The estimated levels of accumulated catalase nanozyme were $25 \mu \mathrm{g} / 1 \times 10^{6}$ cells [8].

\section{Neuro inflammation and nigrostriatal degeneration}

For MPTP intoxication, recipient mice were administered 15-18 mg (based on free-base) MPTP/kg body weight delivered in PBS by four intraperitoneal injections (i.p.), each given every two hours as described $[9,10]$. Two days after the last MPTP injection, mice were injected intra tail vein (i.t.v.) with nanozyme alone or BMM loaded with nanozyme as described above.

For 6-OHDA and LPS intoxications, mice were stereotactically injected with 6-OHDA solution $(10 \mu \mathrm{g}$ 6-OHDA in $0.9 \% \mathrm{NaCl}$ with

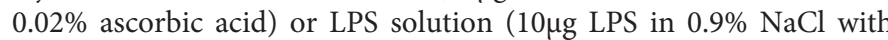
$0.02 \%$ ascorbic acid), respectively, flow rate of $0.1 \mu \mathrm{L} / \mathrm{min}$ into the striatum (AP: +0.5 ; L: -2.0 and DV: $-3.0 \mathrm{~mm}$ ). Two, fourteen, and twenty one day after 6-OHDA intoxication and one day after LPSintoxication animals were injected intratailvein (i.t.v.), orintrajugular vein (i.j.v.) with cell-free and cell-loaded nanozyme as described above.

\section{Pharmacokinetic and biodistribution studies of ${ }^{3} \mathrm{H}$-labeled nanozyme}

A tracer dose of ${ }^{3} \mathrm{H}$-catalase $(4 \mu \mathrm{Ci}, 2 \mu \mathrm{L})$ was mixed with nonlabeled catalase solution $(1 \mathrm{mg} / \mathrm{mL})$ and supplemented with PEI-PEG block copolymer to obtain nanozyme $(\mathrm{Z}=1)$. $\mathrm{BMM}\left(5 \times 10^{6} \mathrm{cells} / \mathrm{ml}\right)$ were loaded with ${ }^{3} \mathrm{H}$-labeled catalase nanozyme as described above. $\mathrm{C} 57 \mathrm{Bl} / 6$ mice with brain inflammation caused by MPTP, 6-OHDA, or LPS intoxications were injected i.t.v. or i.j.v. with i) ${ }^{3} \mathrm{H}$-labeled nanozyme alone, or ii) ${ }^{3} \mathrm{H}$-labeled nanozyme loaded into $\mathrm{BMM}\left(5 \times 10^{6}\right.$ cells in 100 $\mu \mathrm{l}, 4 \mu \mathrm{Ci} /$ mouse). Following various intervals after injection, mice were anesthetized with ketamine/xylazine cocktail, and subjected to transcardial perfusion with ice-cold PBS for 5 minutes following 5 minutes of perfusion with $4 \%$ ice-cold paraformaldehyde in PBS.

Blood samples $(100 \mu \mathrm{l})$ at various times $(0.5-210$ hours $)$ after the nanozyme administration were taken into heparin-coated microhematocrit tubes (Braintree Scientific Braintee, MA), centrifuged for $5 \mathrm{~min}$ at $400 \mathrm{xg}$, and levels of radioactivity were recorded in plasma. Brain, liver, spleen, and kidney were removed, washed in ice-cold saline, blotted and weighed. The organ samples were supplemented with $0.5 \mathrm{ml}$ of tissue solubilizer and then homogenized in a glass tissue homogenizer (Tissue-Tearor, BioSpec Products, Inc., Bartlesville, $\mathrm{OK})$. Each sample was mixed with $5 \mu \mathrm{l}$ of $30 \%$ hydrogen peroxide and incubated at $4^{\circ} \mathrm{C}$ overnight for decolorization. Then, $100 \mu \mathrm{L}$ of the serum or $100 \mu \mathrm{L}$ of the tissue homogenate was placed into $4 \mathrm{ml}$ of a liquid scintillation cocktail, and the radioactivity levels were determined using a Tricarb 4000 (Packard, Meriden, CT). Six mice were used for each treatment group.

The area under the plasma concentration-time curve from time zero to time infinity or AUC was calculated by the trapezoidal rule-extrapolation method [11]. The area from the last data point to time infinity was estimated by dividing the last measured plasma concentration by the terminal rate constant $\left(\lambda_{n}\right)$. Such non compartmental data analysis was used to calculate the area under the moment curve (AUMC), effective elimination half-life $\left(t_{1 / 2}\right)$, mean resident time (MRT) and time-averaged total body clearance (CL).

Brain-serum ratio $(\mu \mathrm{l} / \mathrm{g})=(\% \mathrm{Inj} / \mathrm{g}$ brain $) /(\% \mathrm{Inj} / \mu \mathrm{l}$ serum $)$, where \%Inj was calculated by dividing the total CPM in a sample by the total $\mathrm{CPM}$ given in a single injection were plotted against the exposure time. The slope of the linear portion of the curve represents the steady-state unidirectional influx rate $(\mathrm{Ki})$

\section{Tritiated water exchange of ${ }^{3} \mathrm{H}$-catalase}

To assess the portion of tritium exchanged with body water the following experiment was performed. A tracer dose $(4 \mu \mathrm{Ci})$ of ${ }^{3} \mathrm{H}$-catalase nanozyme was injected i.t.v. into mice. At each sampling time (from 0.5 to 210 hours post dose) the animals were sacrificed 
Citation: Zhao Y, Haney MJ, Mahajan V, Reiner BC, Dunaevsky A, et al. (2011) Active Targeted Macrophage-mediated Delivery of Catalase to Affected Brain Regions in Models of Parkinson's Disease. J Nanomedic Nanotechnol S4:003. doi:10.4172/2157-7439.S4-003

and whole blood samples were immediately centrifuged to obtain plasma. Four mice were used for each time point. Plasma proteins were precipitated with $4 \%$ trichloracetic acid and centrifuged at $1,500 \mathrm{rpm}$ for $10 \mathrm{~min}$ at $4^{\circ} \mathrm{C}$. The obtained solutions were lyophilized and then solubilized in ethanol/water (1:1). The final volume of the solutions was $100 \mu \mathrm{L}$. Gel-permeation chromatography on a Sephadex G25 column with PBS fluid phase was used to estimate the relative amount of a high molecular weight fraction (i.e., ${ }^{3} \mathrm{H}$-catalase) and a low molecular weight fraction (i.e. ${ }^{3} \mathrm{H}_{2} \mathrm{O}$ ). $100 \mu \mathrm{L}$ of each fraction was placed into $4 \mathrm{ml}$ of a liquid scintillation cocktail, and the radioactivity levels were determined using a Tricarb 4000 (Packard, Meriden, CT). The amount of radioactivity associated with the low molecular weight fraction did not exceed $10 \%$ of ${ }^{3} \mathrm{H}$-catalase in the serum at any time point.

\section{Bioimaging and Infrared Spectroscopy (IVIS)}

To reduce fluorescence quenching by fur and auto fluorescence from solid diet, C57Bl/6 mice were shaved and kept on liquid diet for 48 hours prior to the imaging studies. Catalase used for nanozyme preparation was labeled with Alexa Fluor 680 (emission peak of 680) according to manufacturer's instructions. Then, BMM were loaded with Alexa 680 -labeled nanozyme $(1 \mathrm{mg} / \mathrm{ml}$ catalase, $\mathrm{Z}=1)$ and administered i.j.v. to LPS-intoxicated mice $\left(5 \times 10^{6} /\right.$ mouse in $100 \mu \mathrm{l}$ PBS). Saline injected animals were used in the control group. All solutions were injected with the same levels of fluorescence. For background fluorescence level evaluation, all animals were imaged before the injections in the IVIS 200 Series imaging system (Caliper, Xenogen Co., Life Sciences). Injected animals were imaged at various time points ( 1 hour -21 day) post-treatment.

\section{Epifluorescence microscopy}

Fluorescence imaging was used to visualize extravasation of fluorescently tagged, nanozyme-loaded BMM into the brain following inflammation in C57BL/6 mice. Studies were performed in mice with thin-skull cranial windows. Briefly, after being anesthetized, hair, skin and tissue overlying the primary somatosensory cortex was removed. Using a high-torque dental drill (Aseptico) and dental micro blade (Surgistar), the bone was thinned to $10-50 \mu \mathrm{m}$ by hand. A custom made piece of \#0 cover glass (Deckglaser) was then attached to the thinned region using a cyanoacrylate epoxy (Loctite 454, Loctite Corp.) to prevent the bone from regrowing (Figure S1). Immediately following this, a small burr hole was drilled in close proximity to the thin-skull window, and $10 \mu \mathrm{g} / 1.0 \mu \mathrm{L}$ LPS was intracortically injected using a picrospritzer (Picrospritzer III, Parker) to induce neuroinflamation. Twenty four hours after LPS injection, each recipient received $5 \times 10^{6}$ BMM loaded with Alexa 488-labeled nanozyme via i.j.v. injection. Mice were then imaged at 0,4 and 24 hours post injection using epiflourescence microscopy in a moveable objective microscope (Sutter). Time-lapse images were captured at 30 frames/sec.

\section{Immunohistochemical analyses}

To assess the level of inflammation in mouse PD models, microglia activation was evaluated. Mice were sacrificed following various time intervals after intoxication, and perfused according to standard perfusion protocol with PBS, followed by whole body fixation in $4 \%$ PFA in PBS. Healthy non-intoxicated animals were used as controls. Brain, liver and spleen were removed, washed in ice-cold PBS, postfixed in $10 \%$ phosphate-buffered paraformaldehyde, and paraffin embedded. Immunohistochemical analysis was performed in $30 \mu \mathrm{m}$ thick consecutive coronal brain sections. Tissue sections were permeabilized with $0.01 \%$ Triton X-100 in TBS for 30minutes, blocked for 1hour with $10 \%$ normal goat serum (NGS, Vector Laboratories Inc., Burlingame, $\mathrm{CA}$ ), then incubated with primary monoclonal rat anti mouse anti-CD11b antibodies (AbDSerotec, Raleigh, NC) 1:500 dilution), and secondary biotinylated goat anti-rat antibodies (Vector Laboratories, Burlingame, CA, 1:200 dilution). Following incubation with secondary antibodies, VecStain Elite kit (Vector Laboratories) was used according to manufacturer's directions.

\section{Stereologic assays}

The total number of CD11b-positive microglia cells was counted by using the optical fractionator module in Stereo Investigator software (MicroBrightField, Inc., Williston, VT). In agreement with this method, CD11b-positive cells were counted within SN in the same brain hemisphere of every fourth section throughout the entire extent of the SNPc. Each midbrain section was viewed at low power (10x objective), and the $S N p c$ was outlined. Then, the number of stained cells was counted at high power (60x for microglia). Cell counts were obtained from ameboid Mac-1+ cells within the SN and cells per $\mathrm{mm}^{2}$ were calculated.

A
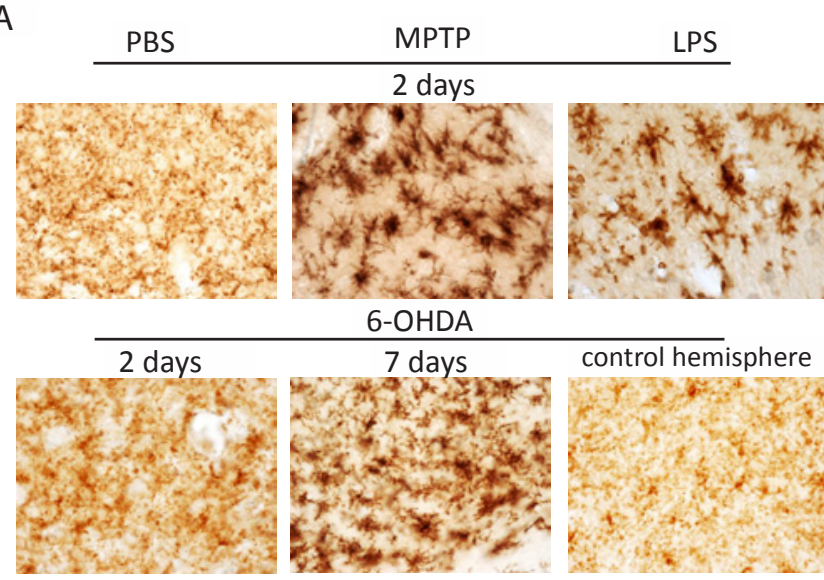

B

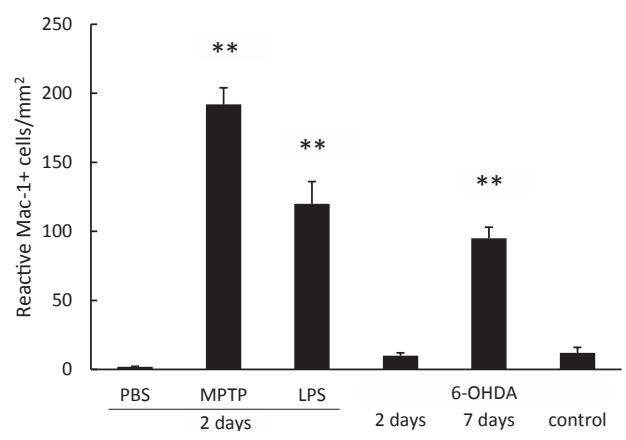

Figure 1: Induction of microglial activation in different PD mouse models

C57Bl/6 mice were injected with MPTP (18mg $/ \mathrm{kg}$, i.p.), LPS $(0.5 \mathrm{mg} / \mathrm{kg}$, i.c.) or $6-\mathrm{OHDA}(0.5 \mathrm{mg} / \mathrm{kg}$, i.c.). Healthy mice with PBS i.c. injections (first bar), and non-injected with 6-OHDA brain hemisphere (last bar) were used in as a control. Two days following intoxication with MPTP or LPS, or seven days after 6-OHDA injections, mice were sacrificed and mid-brain slides were stained for CD11b, a markerfor activated microglia.

A: Representative images of the same experiment. 40x magnification, bright field microscopy.

B: Results from six animals per group demonstrating the treatment mice with MPTP, LPS, and 6-OHDA resulted in significantly increased levels of CD11b compared with non-intoxicated animals (PBS group) or non-injected brain hemisphere in case of 6-OHDA-induced PD model. Statistical significance compared to PBS is shown by asterisk $\left({ }^{* *} p<0.005\right)$. 
Citation: Zhao Y, Haney MJ, Mahajan V, Reiner BC, Dunaevsky A, et al. (2011) Active Targeted Macrophage-mediated Delivery of Catalase to Affected Brain Regions in Models of Parkinson's Disease. J Nanomedic Nanotechnol S4:003. doi:10.4172/2157-7439.S4-003

Page 4 of 8

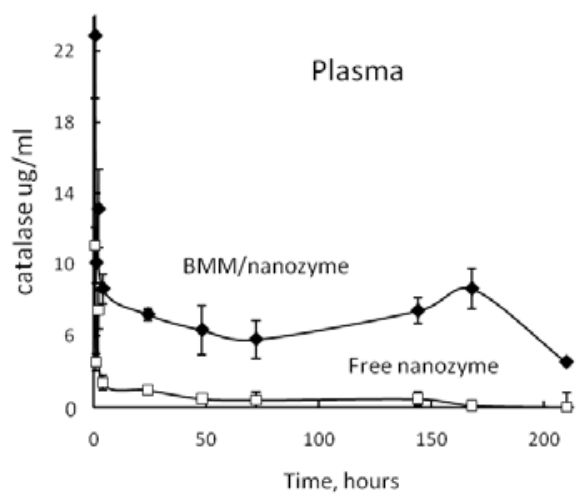

Figure 2: Pharmacokinetics of ${ }^{3} \mathrm{H}$-nanozyme loaded to BMM or cell-free in the blood in MPTP-intoxicated mice. Mice were injected into tail vein with BMM loaded with nanozyme (black diamonds) or cell-free nanozyme (white squares). Following various time intervals after injection, mice were sacrificed and the whole blood samples $(100 \mu \mathrm{l})$ were taken into heparincoated microhematocrit tubes, and immediately centrifuged to obtain plasma. The levels of radioactivity were recorded, and the nanozyme concentration was expressed in $\mu \mathrm{g}$ catalase $/ \mathrm{mL}$ plasma. Each point represents the mean \pm S.E.M. of five mice per time point. The nanozyme organ content was expressed in $\mu \mathrm{g}$ catalase/mg tissue. Five mice were used for each treatment group. BMM loaded ex vivo with nanozyme, and administered into the blood stream play a depot role for the drug, providing considerably greater time of circulation compared to the cell-free catalase nanoformulation.

\begin{tabular}{|l|l|l|}
\hline & nanozyme/BMM & cell-free nanozyme \\
\hline Clearance $(\mathrm{ml} / \mathrm{hr})$ & 2.4 & 6.7 \\
\hline Half-life $(\mathrm{hrs})$ & 3.3 & 2.5 \\
\hline AUC $(\mathrm{ug} \times \mathrm{hrs} / \mathrm{ml})$ & 1465.7 & 518.9 \\
\hline MRT $(\mathrm{hrs})$ & 5.1 & 4.0 \\
\hline Vd $(\mathrm{ml})$ & 12.3 & 26.9 \\
\hline
\end{tabular}

Table 1: The plasma pharmacokinetic parameters of nanozyme administered i.v. in C57BL/6 Mice with MPTP-induced brain inflammation

\begin{tabular}{|l|l|l|}
\hline & nanozyme/BMM & cell-free nanozyme \\
\hline Liver & 246.1 & 53.3 \\
\hline Spleen & 782.4 & 211.5 \\
\hline Kidney & 143.2 & 75.2 \\
\hline Brain & 29.0 & 15.9 \\
\hline
\end{tabular}

Table 2: The AUC $(\mu \mathrm{g} \mathrm{x} \mathrm{h/ml)} \mathrm{nanozyme} \mathrm{administered} \mathrm{i.t.v.} \mathrm{in} \mathrm{C57BI/6} \mathrm{mice} \mathrm{with}$ MPTP-induced brain inflammation.

\section{Statistical analysis}

For all experiments, data are presented as the mean \pm SEM. Tests for significant differences between the groups were done using a one-way ANOVA with multiple comparisons (Fisher's pairwise comparisons) using GraphPad Prism 5.0 (GraphPad software, San Diego, CA, USA). A minimum $p$ value of 0.05 was estimated as the significance level for all tests.

\section{Results}

To evaluate effect of BMM carried nanozyme brain targeting, three murine models of PD were employed: i) intraperitoneal (i.p.) injections of MPTP, ii) intracranial (i.c.) injections of 6-OHDA, and iii) i.c. injections of LPS. Brain inflammation caused by these toxins as manifested by microglia activation was visualized by expression of Mac-1+ ameboid microglia (Figure 1). MPTP and LPS injections induced significant neuroinflammation 48 hours after the intoxication. Microglial activation in animals injected with 6-OHDA developed seven days after intoxication. Noteworthy, inflammation was not detected in non-injected "contralateral" hemisphere (Figure 1). The use of variant models permitted comprehensive assessment of nanozyme cell carriage on drug pharmacokinetics and biodistribution and for disease outcomes.

\section{Pharmacokinetics of BMM-carried nanozyme}

To develop a brain targeted cell-mediated delivery, BMM were loaded ex vivo with the catalase nanozyme and injected via the tail vein (i.t.v.) in C57BL/6 mice with brain inflammation. First, nanozyme plasma concentrations were obtained in MPTP intoxicated mice (Figure 2, black diamonds). Injections of cell-free nanoparticles (without BMM) were used as controls (Figure 2, white squares). BMM substantially increased the circulation time when compared to cellfree nanozyme. A significant amount of nanozyme was detected in the blood for over 160 - 170 hours after injection.

The individual pharmacokinetic parameters for cell-incorporated and free nanozyme in blood were calculated using non-compartmental data analysis (Table 1). The results confirmed that loading to BMM considerably increased the AUC, effective elimination half-life $\left(t_{1 / 2}\right)$, and MRT of the nanozyme. Accordingly, clearance and volume of distribution for macrophage carried nanozyme was decreased from cell-free formulations. This suggested that nanozyme-loaded BMM works as a depot for the enzyme's prolonged circulation and release rates.

\section{Tissue distribution of the BMM carried nanozyme}

The time course of nanozyme accumulation and elimination in the brain, spleen, liver, and kidney administered as free nanoparticle or loaded in BMM at equivalent enzyme concentration are shown in
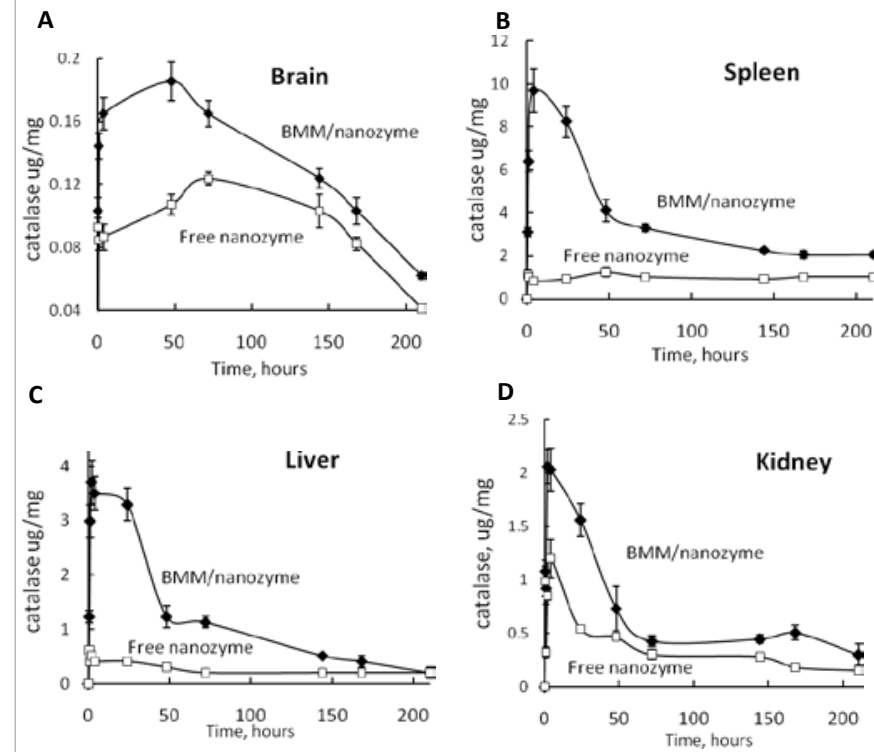

Figure 3: The time course of $3 \mathrm{H}$-nanozyme loaded to BMM and cell-free in the main organs of MPTP-intoxicated mice. Nanozyme loaded into BMM (black diamonds) or cell-free nanozyme (white squares) was administered into tail vein in mice with MPTP injections. At different times after injection, mice were anesthetized, and subjected to transcardial perfusion with $4 \%$ ice-cold paraformaldehyde in PBS. The brain (A), spleen (B), liver (C), and kidney (D) were collected, and the level of radioactivity was recorded. The nanozyme organ content was expressed in $\mu \mathrm{g}$ catalase/mg tissue. Five mice were used for each treatment group. BMM loaded ex vivo with nanozyme, and administered into the blood stream increased drug accumulation levels in the main organs compared to the cell-free catalase nanoformulation. 
Citation: Zhao Y, Haney MJ, Mahajan V, Reiner BC, Dunaevsky A, et al. (2011) Active Targeted Macrophage-mediated Delivery of Catalase to Affected Brain Regions in Models of Parkinson's Disease. J Nanomedic Nanotechnol S4:003. doi:10.4172/2157-7439.S4-003

Figure 3. Macrophage loaded nanozyme increased accumulation of catalase in all tissues (Table 2). The accumulated enzyme was highest in spleen and lower levels were observed in liver then kidney with the lowest levels seen in brain. Notably, the greatest effect of cell carriage on enzyme accumulation levels was in the liver (4.6 fold) and spleen ( 3.7 fold) reflected in AUC values (Table 2). This would be secondary to the natural trafficking pathways of macrophages to the lymphoreticular systems and notably the liver and the added systemic inflammation incurred as a result of MPTP intoxication (Figure S2). All together the data provided clear evidence that loading of nanozyme to BMM increased enzyme delivery to a range of different tissues as well as to the brain that parallel sites of macrophage tissue migration.

\section{Nanozyme transport across the BBB}

To evaluate whether BMM nanozyme carriage affected transport across the $\mathrm{BBB}$, brain/plasma ratios for cell-incorporated and cellfree nanozyme were determined. MPTP-intoxicated mice were i.t.v. injected with ${ }^{3} \mathrm{H}$-nanozyme loaded into the macrophage-carriers, or free nanoparticles. Nanozyme loaded into BMM entered the brain at a faster rate than cell-free nanoparticles (Figure 4). In particular, the unidirectional influx rate $(\mathrm{Ki})$ for $\mathrm{BMM}$-incorporated and free nanozyme were $0.026 \mu \mathrm{l} / \mathrm{gxmin}$ and $0.014 \mu \mathrm{l} / \mathrm{gxmin}$, respectively. This suggested that adoptively transferred BMM facilitates transport of catalase nanoparticles across the BBB during neuroinflammation.

\section{Targeting of nanozyme in BMM to the inflammation site}

To assess BMM targeting to specific sites of neuroinflammation without systemic effect, 6-OHDA-intoxicated mice were employed. Significantly greater nanozyme levels were observed in the brain with lower levels in kidney, spleen, liver, and lungs. This was recorded in mice that recieved i.c. 6-OHDA injection compared to MPTP intoxicated animals (Figure 5). No effect of peripheral inflammation caused by i.p. MPTP injections on macrophage-free nanozyme distribution was found with the exception of the brain (Figure 5). Thus, we concluded that 6-OHDAcan model cell-mediated drug delivery specifically to brain subregions.

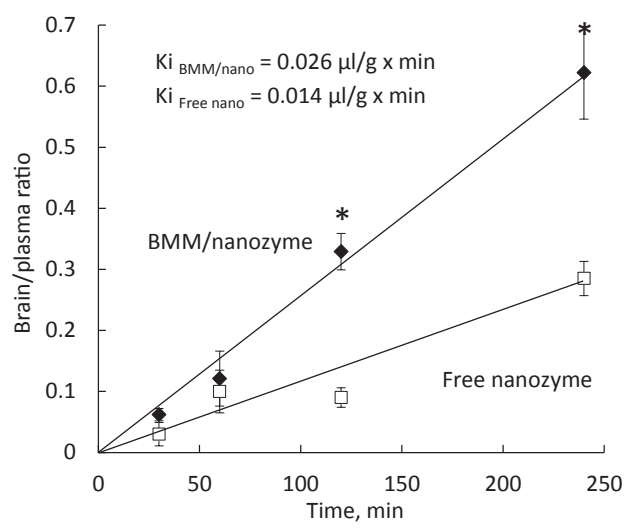

Figure 4: Macrophages facilitate transport of nanozyme across the BBB in murine model of PD. ${ }^{3} \mathrm{H}$-catalase nanozyme loaded into BMM (black diamonds) or cell-free nanoparticles (white squares) were injected i.t.v. in MPTP-intoxicated mice. In parallel experiment, BMM loaded with thye same amount of nanozyme were injected to healthy animals (crosses). Blood and brain samples at various time points were taken, and radioactivity levels were recorded. Results from $\mathrm{N}=6$ mice per group ( \pm SEM) demonstrating significant increase in cell-incorporated nanozyme transport across the BBB compared to cell-free nanoparticles $(*)$ and transport of BMM-incorporated nanozyme in healthy mice (\#). Statistical significance is shown by asterisk $(p<0.05)$.
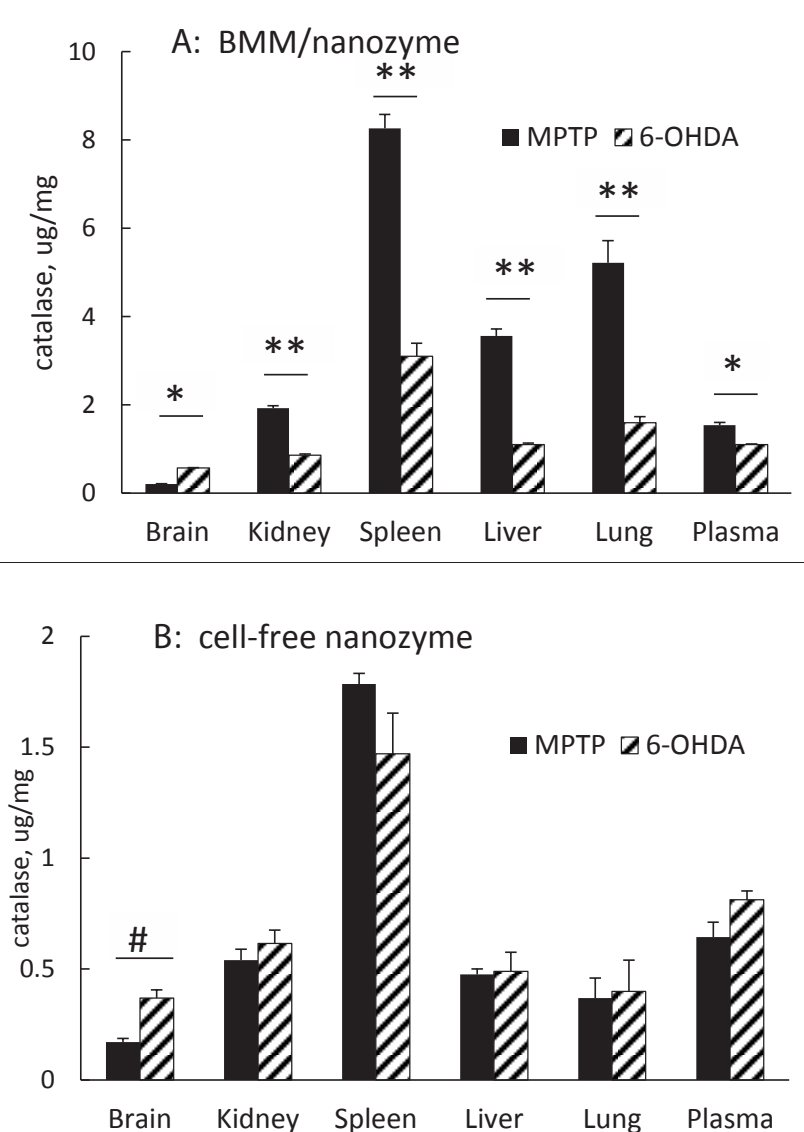

Figure 5: Targeting of nanozyme loaded into BMM to the sites with inflammation. Mice were intoxicated with i.p. MPTP injections (solid bars) or i.c. 6-OHAD injections (diagonal pattern bars) to produce PD model. Seven

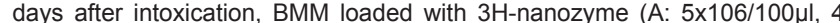
$\mu \mathrm{Ci} /$ mouse) or cell-free $3 \mathrm{H}$-labelled nanozyme alone (B) were injected intra tail vein. Four hours following nanozyme administration, mice were anesthetized perfused, the main were collected, and the level of radioactivity was assessed Results from $\mathrm{N}=6$ mice per group ( \pm SEM) demonstrated significant increase of brain nanozyme levels in mice subjected to i.c. 6-OHDA injections compared to mice with i.p. MPTP injections (A). Contrary, lower nanozyme levels were recorded in kidney, spleen, liver and lungs in mice with 6-OHDA intoxications. This indicates that inflammation in peripheral organs caused by i.p. MPTP injections resulted in redistribution of the cell carriers loaded with nanozyme. No effect of peripheral inflammation was found with macrophagefree nanozyme administered without cell-carriers with the brain exception (B). Statistical significance is shown by asterisk $(*, p<0.05)$, and double asterisk $(* *, p<0.005)$.

The localized injection of 6-OHDA provides an opportunity to analyze the targeting of cell-mediated drug delivery into affected brain subregions because the uninjected contralateral brain hemisphere provides an internal control. The obtained data clearly indicate that macrophages increased nanozyme transport across the BBB only in the ipsilateral hemisphere. Thus, brain/serum ratio in the brain hemisphere with 6-OHDA lesion for BMM-carried nanozyme was significantly greater than in contralateral hemisphere as observed on day seven after intoxication (Figure 6, black bars), at time when microglial activation was readily seen (Figure 1). In contrast, there was no difference between hemispheres in levels of BMM carried nanozyme on the second day after intoxication (Figure 6, white bars). This was likely due to the fact that inflammation was not yet developed (Figure 1). Noteworthy, only subtle effects of 6-OHDA injections on the cell- 
Citation: Zhao Y, Haney MJ, Mahajan V, Reiner BC, Dunaevsky A, et al. (2011) Active Targeted Macrophage-mediated Delivery of Catalase to Affected Brain Regions in Models of Parkinson's Disease. J Nanomedic Nanotechnol S4:003. doi:10.4172/2157-7439.S4-003

Page 6 of 8

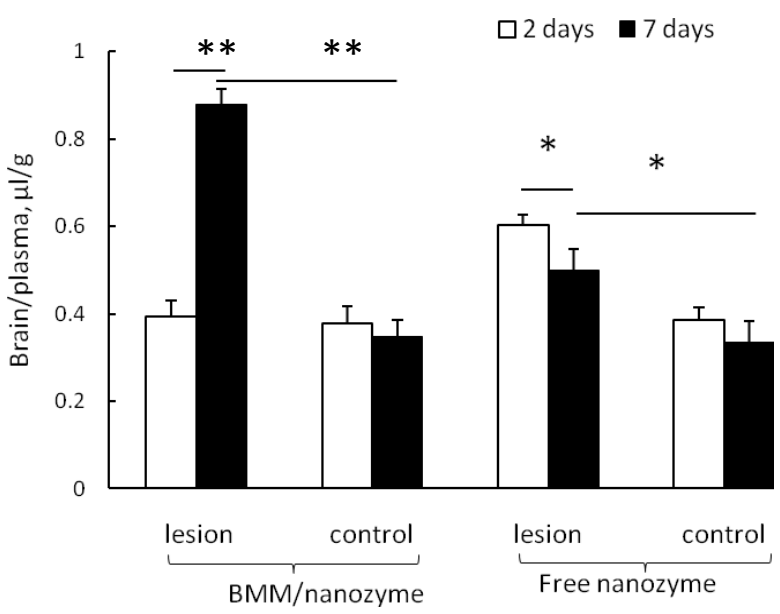

Figure 6: BMM facilitate transport of incorporated nanozyme across the BBB toward brain inflammation. ${ }^{3} \mathrm{H}$-catalase nanozyme loaded into BMM or cell-free nanoparticles were i.t.v. injected in 6-OHDA intoxicated C57BL/6 mice on second (white bars), or seventh (black bars) day after intoxication. Blood and brain samples were taken four hours after the administration, and radioactivity levels were recorded. Results from $\mathrm{N}=6$ mice per group (mean \pm SEM) demonstrate significant increase in cell-incorporated nanozyme transport across the BBB in the hemisphere with 6-OHDA injection. No effect in healthy hemisphere was found. Statistical significance is shown by asterisk $\left({ }^{*} p<0.05,{ }^{* *} p<0.005\right)$.

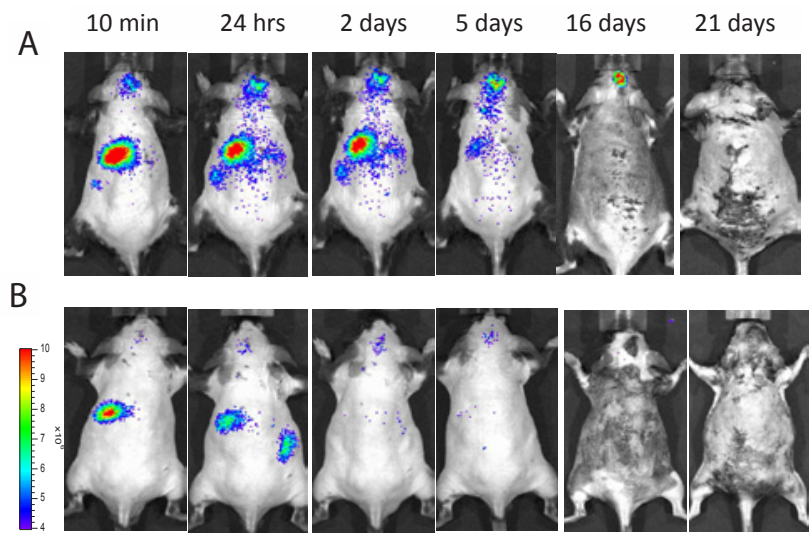

Figure 7: Biodistribution of BMM-carried nanozymes in mice with PD model by IVIS. LPS-intoxicated (i.c., $0.5 \mathrm{mg} / \mathrm{kg})(\mathbf{A})$, or healthy C57BL/6 mice were i.j.v. injected with Alexa Fluor 680-labeled nanozyme loaded to BMM $\left(5 \times 10^{6} \mathrm{cells} /\right.$ mouse). Representative images from $\mathrm{N}=4$ mice per group (dorsal planes) taken at various time points demonstrate prolonged circulation time of nanozyme in the mice with brain inflammation (A) compared to the healthy animals (B). Redistribution of nanozyme from peripheral organs to the brain with inflammation was detected on the second week after adoptive cell transfer in LPS-intoxicated animals (A).

free nanozyme transport were detected. The slightly elevated levels of free nanoparticles in the ipsilateral hemisphere on the second day after intoxication, is likely due to $\mathrm{BBB}$ break down caused by i.c. injections of 6-OHDA that was not yet restored at this early time point.

Route of nanozyme administration affects enzyme bioavailability to the brain

The route of administration was next investigated for the amount of cell-incorporated nanozyme brain delivery. Two intravenous routes, intrajugular vein (i.j.v.), and intra-tail vein (i.t.v.) injections were evaluated in this study. I.j.v. injections provided substantially greater levels of the nanozyme delivered to the brain compared to i.t.v. injections. Thus, percent of the injected dose of cell-incorporated nanozyme in the brain in 6-OHDA-intoxicatedmice upon i.j.v. and i.t.v. administration was 3.4 and $0.2 \%$, respectively, on the tenth day after cell transfer. This suggested that i.j.v. administration allowed a portion of the cells bypass the liver homing the brain inflammation sites.

\section{Biodistribution of BMM-carried nanozymes visualization by IVIS}

To visualize distribution of cell-loaded nanozyme, IVIS studies were conducted in mice with LPS-induced brain inflammation. BMM loaded with fluorescently-labeled nanozyme were administered i.j.v. to C57BL/6 mice with brain inflammation (Figure 7A). Non-intoxicated mice were used as controls (Figure 7B). Fluorescent and light images of dorsal (Figure 7) planes of the injected animals were taken at various times. Striking difference in circulation time of nanozyme in the mice with brain inflammation (Figure 7A) compared to noninflamed animals (Figure 7B) was demonstrated. Nanozyme was detected over 16-20 days after nanozyme-BMM transferin the brain with inflammation, and only for 1-2 days in the non-inflamed animals. Furthermore, redistribution of nanozyme from peripheral organs to the brain with inflammation was detected on the second week after BMM transfer in LPS-intoxicated animals (Figure 7A). This provides additional evidence that BMM hold considerable portion of nanozyme, accomplishing targeted drug delivery to the tissues with inflammation.

\section{$\mathrm{BMM}$ translocation across the $\mathrm{BBB}$}

Using IVIS imaging does not allow to distinguish between BMM loaded with fluorescent-labeled nanozyme that are present in the blood stream or in the brain parenchyma. To eliminate this factor,
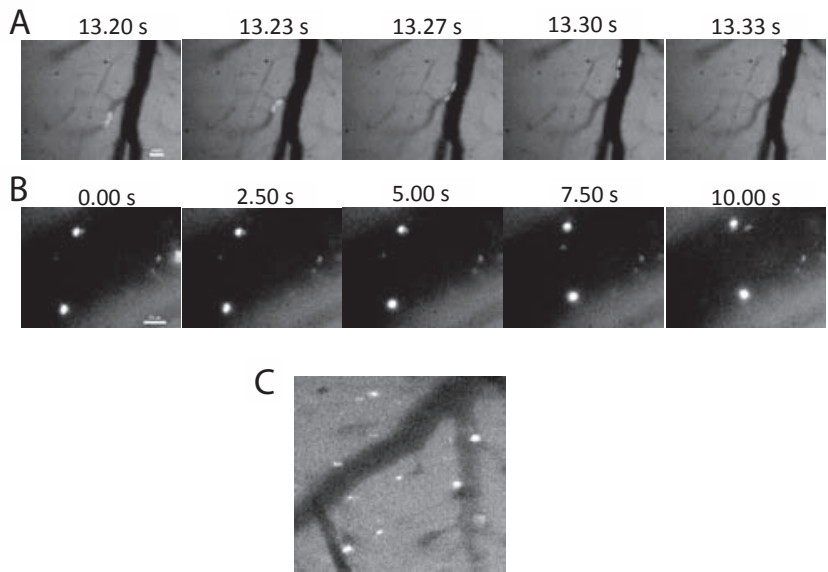

Figure 8: In vivo epifluorescence imaging of translocation of BMM loaded with catalase nanozyme across the BBB in a neuroinflamation mouse model. C57BL/6 mice with cranial windows were i.c. injected with 10ug LPS to produce inflammation in the brain area close to the window. Twenty four hours after intoxication, BMM loaded with Alexa 488-labeled nanozyme were adoptively transferred via i.j.v., and images A: immediately after administration; B: 4 hours and C: 24 hours after the injection were taken Macrophages carrying fluorescently labeled nanozyme first seen moving fast along the microvessels $(\mathbf{A})$, then slow down and adhere to the endothelial wall (B), and finally, were stationary and appeared to have translocated across the BBB into the parenchyma(C). Bar: $20 \mu \mathrm{m}$. 
epifluorescence in vivo microscopy in mice with thin-skull cranial windows was utilized. Mice with LPS-induced brain inflammation received BMM loaded with fluorescently-labeled nanozyme by i.j.v. injection and were imaged at various times. Loaded BMM were first seen to move along the microvessels (immediately following injection, Figure 8A, Media 1), then adhered to the endothelial wall (4hrs post injection, Figure 8B, Media 2), and finally, were stationary and appeared to have translocated across the BBB into the parenchyma (24hrs post injection, Figure 8C).

\section{Discussion}

Our works focus on the development a targeted cell-mediated delivery of therapeutic polypeptides to the brain to attenuate neuroinflammation and produce neuro protection for PD. Indeed, due to the relatively local neuronal degeneration, $\mathrm{PD}$ is a particular example where targeted drug delivery is required. Using macrophages as drug delivery systems takes advantage of its natural carriage, storage, mobility and secretory capacities. Indeed, neuroinflammation in PD is seen commonly. Blood borne macrophages can affect BBB breakdown during neurodegenerative processes in disease [12-15]. Interestingly, prior reports indicate that monocytes traffic primarily between adjacent endothelial cells, i.e. paracellularily through the junctional complexes [16,17]. Thus, monocyte-macrophages can readily migrate toward inflammation sites and show inherent chemotaxic and diapedesis activities [18].Therefore, brain inflammation that develops in PD patients may support targeted delivery of catalase by monocytes to the SNPc. Taking advantage of such pathobiologic events, we have developed macrophages as a drug transporter. In the current work, we demonstrated that BMM loaded ex vivo with an antiinflammatory polypeptide, catalase, and administered into the blood stream creates a depot of the drug in tissues, providing considerably greater times in circulation and bioavailability to the main organs, including brain, kidney, spleen, and liver, compared to the cell-free catalase nanoformulation. Furthermore, in addition to the increased doze of nanozyme in the brain, the cell-carriers improved the BBB permeability, increasing brain/plasma ratio of cell-incorporated nanozyme compared to the free drug.

Noteworthy, inflammation induced in the brain (or peripheral organs) attracted macrophages loaded with nanozyme resulting in the increased drug accumulation in these tissues. In contrast, effect of inflammation on cell-free nanozyme accumulation levels was negligible. In this respect, we concluded that probably, i.c. intoxication with 6-OHDA and LPS may be more appropriate PD models for cellmediated drug delivery evaluations than i.p. MPTP injections that caused significant inflammation process in the peripheral organs. High enzyme levels administered in macrophages and accumulated in the lymphoreticular system may cause toxicity that differs from those imposed by chemicals in classical delivery matrices. Thus, the toxicology of cell-mediated drug formulations should be addressed in further investigations as it is important for their translation to clinic. Nevertheless, redistribution of the portion of nanozyme initially deposited in the peripheral organs, liver, spleen and kidney, to the brain inflammation area was demonstrated by IVIS. Overall, this indicates that BMM retain a significant portion of nanoparticles transporting them across the BBB to sites of disease and inflammation.

Remarkably, all three proposed mechanisms of cell-mediated carriage of catalase nanozyme were confirmed; i) facilitated transport across the BBB ("Trojan Horse" effect), ii) increased enzyme blood circulation time (a "depot" effect) and iii) catalase redistribution from peripheral organs, liver and spleen to the brain. Noteworthy, the redistribution and collecting of catalaseat the brain affected regions was especially noticeable in IVIS studies in living mice over two weeks after the cell administration. We hypothesized that two processes may be responsible for this effect: i) the cell-carriers with loaded nanozyme that populated the peripheral organs at the beginning were recruited later to the inflammation site delivering catalase to the brain; ii) nanozyme brought by the cell-carriers and released first in the liver, spleen, and kidneys was taken later by peripheral lymphocytes in these organs and then delivered to the brain inflammation site. Overall, the impact of cell-carriers on nanozyme brain transport is substantial. Administration of cell-incorporated nanozyme through i.j.v. injections allows cell-carriers to bypass the liver at least in the first circle and results in considerably greater nanozyme accumulation in the affected brain. Using cell-carriers for brain drug delivery may open a new perspective of active delivery of drugs for treatment of PD. Furthermore, prolonged and sustained release of catalase from macrophages in the brain may also be beneficial for treatment of chronic inflammation manifested in PD patents. A more comprehensive understanding these issues is crucial for the future development of drug delivery system based on cell-carriers as well as its role in a broader range of disorders of the nervous system.

\section{Acknowledgments}

This study was supported by the National Institutes of Health grants 1R01 NS057748 (to EVB), 2R01 NS034239, 2R37 NS36126, P01 NS31492, P20RR 15635, P01 MH64570, P01 NS43985 (to HEG), RR021937 (to AVK), 1R01 NS070190 (to RLM), and Russian Ministry of Science and Education grants 02.740.11.5231 and 11.G34.31.0004 (to AVK).

\section{References}

1. Batrakova EV, Li S, Reynolds AD, Mosley RL, Bronich TK, et al. (2007) A macrophage-nanozyme delivery system for Parkinson's disease. Bioconjug Chem 18: 1498-1506.

2. Brynskikh AM, Zhao Y, Mosley RL, Li S, Boska MD, et al. (2010) Macrophage delivery of therapeutic nanozymes in a murine model of Parkinson's disease. Nanomedicine (Lond) 5: 379-396.

3. Zhao Y, Haney MJ, Klyachko NL, Li S, Booth SL, et al. (2011) Polyelectrolyte complex optimization for macrophage delivery of redox enzyme nanoparticles. Nanomedicine (Lond) 6: 25-42.

4. Dou H, Destache CJ, Morehead JR, Mosley RL, Boska MD, et al. (2006) Development of a macrophage-based nanoparticle platform for antiretroviral drug delivery. Blood 108: 2827-2835.

5. Vinogradov S, Bronich T, Kabanov A (1998) Self-assembly of polyaminepoly(ethylene glycol) copolymers with phosphorothioate oligonucleotides. Bioconjug Chem 9: 805-812.

6. Batrakova EV, Han H, Miller D, Kabanov A (1998) Effects of pluronic P85 unimers and micelles on drug permeability in polarized BBMEC and Caco-2 cells. Pharm Res 15: 1525-1532.

7. Batrakova EV, Vinogradov SV, Robinson SM, Niehoff ML, Banks WA et al. (2005) Polypeptide point modifications with fatty acid and amphiphilic block copolymers for enhanced brain delivery. Bioconjug Chem 16: 793-802.

8. Haney MJ, Zhao Y, Li S, Higginbotham SM, Booth SL, et al. (2011) Cellmediated transfer of catalase nanoparticles from macrophages to brain endothelial, glial and neuronal cells. Nanomedicine (Lond).

9. Benner EJ, Mosley RL, Destache CJ, Lewis TB, Jackson-Lewis V, et al. (2004 Therapeutic immunization protects dopaminergic neurons in a mouse model of Parkinson's disease. Proc Natl Acad Sci USA 101: 9435-9440.

10. Jackson-Lewis V, Przedborski S (2007) Protocol for the MPTP mouse model of Parkinson's disease. Nat Protoc 2: 141-151.

11. Gibaldi M, Perrier D, Swarbrick J (1982) Pharmacokinetics, Marcel Dekker Inc., New York 409-416. 
Citation: Zhao Y, Haney MJ, Mahajan V, Reiner BC, Dunaevsky A, et al. (2011) Active Targeted Macrophage-mediated Delivery of Catalase to Affected Brain Regions in Models of Parkinson's Disease. J Nanomedic Nanotechnol S4:003. doi:10.4172/2157-7439.S4-003

Page 8 of 8

12. Anthony DC, Bolton SJ, Fearn S, Perry VH (1997) Age-related effects of interleukin-1 beta on polymorphonuclear neutrophil-dependent increases in blood-brain barrier permeability in rats. Brain 120: 435-444.

13. Anthony DC, Blond D, Dempster R, Perry VH (2001) Chemokine targets in acute brain injury and disease. Prog Brain Res 132: 507-524.

14. Blamire AM, Anthony DC, Rajagopalan B, Sibson NR, Perry VH, et al. (2000) Interleukin-1beta -induced changes in blood-brain barrier permeability apparent diffusion coefficient, and cerebral blood volume in the rat brain: a magnetic resonance study. J Neurosci 20: 8153-8159.

15. Persidsky Y, Ghorpade A, Rasmussen J, Limoges J, Liu XJ, et al. (1999)
Microglial and astrocyte chemokines regulate monocyte migration through the blood-brain barrier in human immunodeficiency virus-1 encephalitis. Am J Pathol 155: 1599-1611.

16. Pawlowski NA, Kaplan G, Abraham E, Cohn ZA (1988) The selective binding and transmigration of monocytes through the junctional complexes of human endothelium. J Exp Med 168: 1865-1882.

17. Lossinsky AS, Shivers RR (2004) Structural pathways for macromolecular and cellular transport across the blood-brain barrier during inflammatory conditions. Review. Histol Histopathol 19: 535-564.

18. J. Kuby (1994) Immunology, Freeman, WH. and Co., New York 